\title{
Weak deflection angle by asymptotically flat black holes in Horndeski theory using Gauss-Bonnet theorem
}

\author{
W. Javed, ${ }^{1, *}$ J. Abbas, ${ }^{2, \dagger}$ Y. Kumaran, ${ }^{3, \ddagger}$ and A. Övgün ${ }^{3, \S}$ \\ ${ }^{1}$ Division of Science and Technology, University of Education, Township-Lahore, Pakistan \\ ${ }^{2}$ Department of Mathematics, University of Education, \\ Township, Lahore-54590, Pakistan. \\ ${ }^{3}$ Physics Department, Arts and Sciences Faculty, Eastern Mediterranean University, Famagusta, North Cyprus via Mersin 10, Turkey.
} (Dated: July 28, 2020)

\begin{abstract}
The principal objective of this project is to investigate the gravitational lensing by asymptotically flat black holes in the framework of Horndeski theory in weak field limits. To achieve this objective, we utilize the Gauss-Bonnet theorem to the optical geometry of asymptotically flat black holes and applying the Gibbons-Werner technique to achieve the deflection angle of photons in weak field limits. Subsequently, we manifest the influence of plasma medium on deflection of photons by asymptotically flat black holes in the context of Horndeski theory. We also examine the graphical impact of deflection angle on asymptotically flat black holes in the background of Horndeski theory in plasma as well as non-plasma medium.
\end{abstract}

PACS numbers: 04.40.-b, 95.30.Sf, 98.62.Sb

Keywords: Weak gravitational lensing; Deflection of light; Black hole; Deflection angle; Horndeski theory; GaussBonnet theorem

\section{INTRODUCTION}

The anecdote of a falling apple fostering Newtonian gravity has been imparted on generations since the year 1666. For centuries from then, physicists have believed that the gravitational force is related to the ratio of the product of the interacting masses and the square of their separation through a proportionality constant. This 'Gravitational' constant was given an accurate value via the Cavendish experiment. In 1910s, Einstein's general theory of relativity transpired suggesting a finite, spherical universe in which the Gravitational constant, $G$ was discerned to exhibit a dependence on mass distribution and size of the universe, so as to account for the inertial forces [1].

A decade later, Hubble's observations confirmed the Big Bang Theory, essentially establishing that the universe was expanding, and potentially infinite. The extent of the universe that can be observed is limited to a maximum distance determined by the time that light takes to reach Earth from the observable edge. In other words, mass distribution and the size of the universe changes with time, and hence, so does the gravitational constant, $G[2,3]$. This discrepancy led to the speculation that the effect of $G$ could rather be a scalar field, than a constant number. According to Einstein's formulation, the so-called metric field contains the influence of gravity, and mathematically known as a tensor. Therefore, the idea of consolidating a scalar field due to mass distribution with the metric is called as the scalar-tensor theory [4-6].

One such generic gravitational scalar-tensor theory is the Horndeski theory [7]. Defined for a four-dimensional spacetime, the scalar field is incorporated as a new degree of freedom to formulate the Lagrangian of the system, begetting second-order field equations of motion. This notion is inspired from the Lovelock theory of gravity: relaxing Lovelock's assumptions not only exercises the scalar-tensor theory, but also extends Einstein's theory of gravity.

The kinetic term of the Lagrangian characterizes the quadratic derivative of the field and depends on the Lovelock tensor - which is proportional to the Einstein tensor, $G_{\mu \nu}$ - encompassing non-minimal coupling between the scalar field, $\varphi$ and curvature [8]. Hence, the action principle for a 4-D spacetime $(n=4)$ becomes [9, 10]:

$$
I\left[g_{\mu \nu}, \varphi\right]=\int \sqrt{-g} d^{4} x\left[k(R-2 \Lambda)-\frac{1}{2}\left(\alpha g_{\mu \nu}-\eta G_{\mu \nu}\right) \nabla^{\mu} \varphi \nabla^{\nu} \varphi\right]
$$

\footnotetext{
*Electronic address: wajiha.javed@ue.edu.pk; wajihajaved84@yahoo.com

${ }^{\dagger}$ Electronic address: jameelaabbas30@gmail.com

‡Electronic address: y.kumaran13@gmail.com

$\S$ Electronic address: ali.ovgun@emu.edu.tr; URL: https : / / www.aovgun.com
} 
where, $k \equiv 1 / 16 \pi G$. Here, the first term accounts for the scalar field with non-minimal coupling for matter owing to the Ricci scalar, $R$, and the second term accounts for the Einstein-Hilbert action for gravity owing to the cosmological constant, $\Lambda$. Values of the parameters $\alpha$ and $\eta$ are governed by the positive energy density of matter field.

The above equation is the reduced form of the action analyzed with standard matter, matter fields and nonstandard scalar field [10]: implementing assumptions - the geometry is static, spherically symmetric and homogeneous with the scalar and metric fields obeying this symmetry in an asymptotically flat space-time - renders this equation as the limiting case of Horndeski theory. Eq. 1 is the foundation of the ensuing work.

Gravitational lensing is a consequence of general relativity, where the gravitational force due to a massive object bends the paths of light originating from distant objects lying behind it, which is used to understand galaxies, dark matter, dark energy and the universe [1]. Various authors studied the gravitational lensing by black holes, wormholes, cosmic strings and other objects since the first gravitational lensing observation by Eddington [11-37].

In 2008, Gibbons and Werner cleverly showed that there is a alternative way to obtain weak deflection angle for asymptotically flat optical spacetimes using the Gauss-bonnet theorem [38]. Afterwards, Werner was managed to derive the weak deflection angle of stationary spacetimes using the Gauss-bonnet theorem [39]. Note that both of them were used the way that the source and receiver are located at asymptotic regions. Next, Ishihara et al. showed that it is also possible to use this method for finite-distances (large impact parameter cases) [40]. Then, Crisnejo and Gallo showed that plasma medium deflects photons [41]. For more recent works, one can see [42-82].

The purpose of this work is to study the deflection angle by black holes in a Horndeski theory using the GaussBonnet theorem to test the validity of this modified gravity theory. For comparison, we consider the notion of the deflection angle of massive particles in a plasma medium from a black hole. Our main aim is to check the effects of Horndeski theory on the weak deflection angle.

This paper is organized as follows: In section 2, we review some basic concepts about asymptotically flat $\mathrm{BH}$, then we compute the Gaussian optical curvature for deflection angle and calculate the deflection angle by using GBT. In section 3, we calculate the deflection angle for in a plasma medium. The last section comprises of concluding remarks.

\section{CALCULATION OF PHOTON LENSING FOR FOR ASYMPTOTICALLY FLAT BLACK HOLES}

Here we will show that the inclusion of a cosmological term in the action allows finding a new asymptotically locally flat black hole. In this case we will consider that the matter sector is given only by the kinetic term of the scalar field which is constructed with the Einstein tensor, namely we shall consider the action with $\alpha=0$. Thus, the action reduces to

$$
I\left[g_{\mu \nu}, \varphi\right]=\int \sqrt{-g} d^{4} x\left[k(R-2 \Lambda)+\frac{\eta}{2} G_{\mu \nu} \nabla^{\mu} \varphi \nabla^{\nu} \varphi\right]
$$

The field equation allows a first integral which introduces an integration constant. If we set it to zero, we find that for $K \neq 0$, the following metric defines a solution of the system [9]:

$$
d s^{2}=-H(r) d t^{2}+\frac{15\left(\Lambda r^{2}-2 K\right)^{2}}{K} \frac{d r^{2}}{H(r)}+r^{2} d \Sigma_{K, 2}^{2}
$$

where $d \Sigma_{K, 2}^{2}=d \theta^{2}+\sin ^{2} \theta d \varphi^{2}$ and

$$
H(r)=\left(60 K^{2}-20 \Lambda K r^{2}+3 \Lambda^{2} r^{4}\right)-\frac{\mu}{r}
$$

If $\Lambda$ disappears, the scalar field vanishes and the solution turns into the topological Schwarzschild solution in a flat space-time, representing a black hole only in a spherically symmetrical scenario [9]. By taking $\Lambda=0$ :

$$
d s^{2}=-H(r) d t^{2}+\frac{60 K}{H(r)} d r^{2}+r^{2} d \Sigma_{K, 2}^{2}
$$

and

$$
H(r)=60 K^{2}-\frac{\mu}{r}
$$

Here, $\mu$ is the integration constant and can be explicate as the black hole mass. 
Now to acquire the null geodesics $\left(d s^{2}=0\right)$, the $\mathrm{BH}$ optical spacetime can be simply written in equatorial plane $\theta=\frac{\pi}{2}$ :

$$
d t^{2}=\frac{60 K}{H(r)^{2}} d r^{2}+\frac{r^{2}}{H(r)} d \varphi^{2}
$$

along with optical metric $\hat{g}_{a b}^{o p t}=\frac{g_{a b}}{\left(-g_{t t}\right)}$, according to the Fermat principle, the geodesics are spatial rays of light.

The Gaussian optical curvature can be determined by allowing use of the description for the two-dimensional optical metric 7

$$
\mathcal{K}=\frac{R_{\text {icciScalar }}}{2}
$$

in which $R$ for optical metric is the Ricciscalar. Following the computation of non-zero Christoffel symbols, we obtain the following equation particularly for the Gaussian optical curvature of the optical metric 7

$$
\mathcal{K}=-\frac{K \mu}{r^{3}}+\frac{\mu^{2}}{80 K r^{4}}+\mathcal{O}\left(r^{-5}\right)
$$

Let us recall the GBT for a two dimensional manifold. In this regard, we consider a regular domain $D_{R}$ aligned by 2-dimensional surface $S$ with Riemannian metric $\hat{g}_{i j}$, along with its piece-wise smooth boundary $\partial D_{R}=\gamma_{g} \cup C_{R}$, then GBT permits a connection among the geometry and topology in terms of the subsequent relation [38]

$$
\iint_{D_{R}} \mathcal{K} d S+\oint_{\partial D_{R}} \hat{k} d \sigma+\sum_{j} \tilde{\alpha}_{j}=2 \pi \mathcal{X}\left(D_{R}\right) .
$$

where $\mathcal{K}$ is the Gaussian optical curvature, $\tilde{\alpha}_{j}$ is the exterior angle at the $j^{\text {th }}$ vertex and $\sigma$ is the line element along the boundary $D_{R}$. Let $\gamma$ be a smooth curve in the same domain. Thus, $\dot{\gamma}$ comes to be the unit speed vector [38]. It is well known that for regular domain the Euler characteristic $\mathcal{X}_{D_{R}}=1$, while $\hat{k}$ is termed as a geodesic curvature and is defined as

$$
\hat{k}=g^{o p t}\left(\nabla_{\dot{\gamma}} \dot{\gamma}, \ddot{\gamma}\right)
$$

having the unit speed condition $g^{\text {opt }}(\dot{\gamma}, \dot{\gamma})=1$, where $\ddot{\gamma}$ is the unit acceleration vector perpendicular to $\dot{\gamma}$. In the case of $R \rightarrow \infty$, the respective jump angles are taken as $\pi / 2$ (in short, the sum of angles corresponding to the observer and the source: $\tilde{\alpha}_{O}+\tilde{\alpha}_{S} \rightarrow \pi$ ).

Using the fact that, from geodesic there is a zero contribution i.e. $\hat{k}\left(\gamma_{\tilde{g}}\right)=0$, we shall pursue a contribution by virtue of the curve $C_{R}$ and this contribution can be computed as

$$
\hat{k}\left(C_{R}\right)=\left|\nabla_{\dot{C}_{R}} \dot{C}_{R}\right| \text {. }
$$

Let us consider $C_{R}:=r(\varphi)=R=$ const, while $R$ endows the distance from the coordinate origin. The radial component of the geodesic curvature states as

$$
\left(\nabla_{\dot{C}_{R}} \dot{C}_{R}\right)^{r}=\dot{C}_{R}^{\varphi}\left(\partial_{\varphi} \dot{C}_{R}^{\varphi}\right)+\Gamma_{\varphi \varphi}^{r}\left(\dot{C}_{R}^{\varphi}\right)^{2}
$$

Doing the analogous identifications among the frequencies, energy and mass, which implies the form invariant quantity

$$
\lim _{R \rightarrow \infty}\left[\hat{k} \frac{d \sigma}{d \varphi}\right]_{C_{R}}=1
$$

ensuing in this manner the form invariant deflection angle [38]

$$
\tilde{\alpha}=-\int_{0}^{\pi} \int_{b / \sin \varphi}^{\infty} \mathcal{K} d S
$$

where $b$ is the impact parameter. This equation inscribe the global impact on the lensing of particles on account of the fact that one has to integrate over the optical domain of integration outside the enclosed mass.

Now, by using Eq. 9 into Eq. 15 we obtain the deflection angle for flat black holes in Horndeski theory.

$$
\tilde{\alpha}=\frac{2 K \mu}{b}+\frac{\mu^{2} \pi}{320 K b^{2}}+\mathcal{O}\left(\mu^{3}\right) .
$$




\section{A. Graphical Analysis}

This segment is dedicated to review the graphical impact of deflection angle $\tilde{\alpha}$ on asymptotically flat BHs. Additionally, illustrate the physical eminence of these graphs to examine the influence of curvature constant $K$ and impact parameter $b$ on deflection angle by analyzing the stable and unstable state of $\mathrm{BH}$.

\section{Deflection angle versus Impact parameter $b$}

This subsection gives the analysis of deflection angle $\tilde{\alpha}$ with impact parameter $b$ for different values of curvature constant $K$, for fixed $\mu=2$.
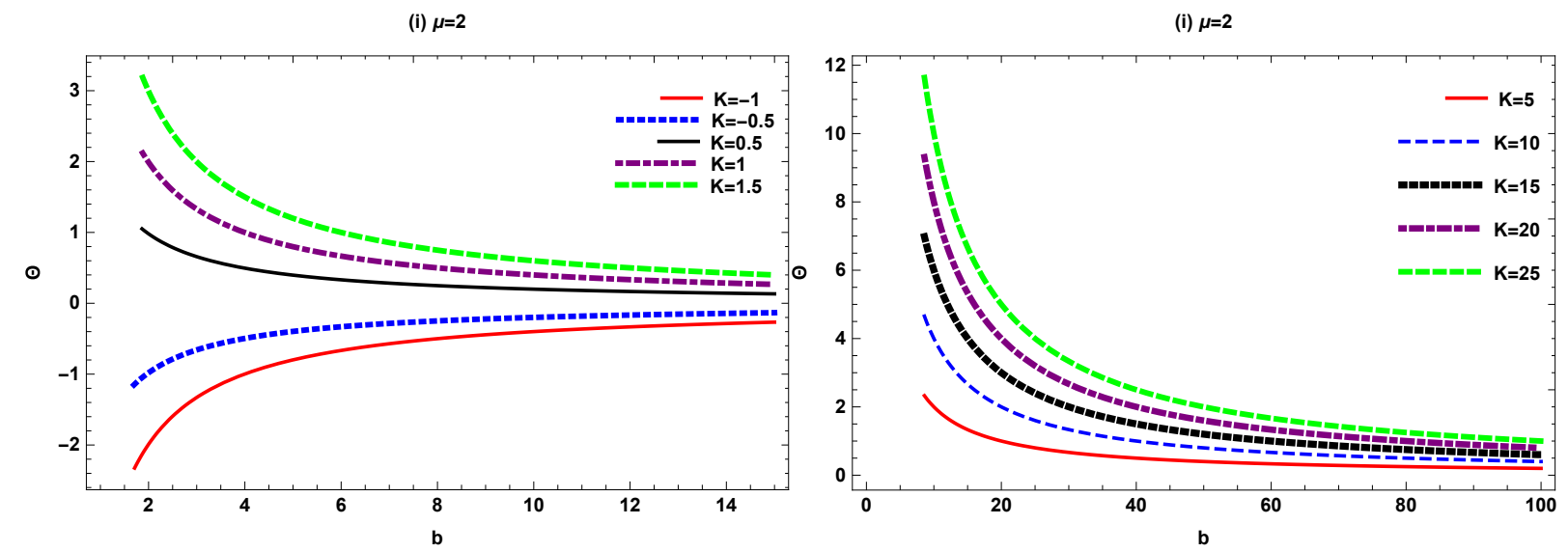

Figure 1: $\tilde{\alpha}$ versus $b$.

- Figure 1 demonstrates the influence of $\tilde{\alpha}$ w.r.t $b$ for different values of $K$. One can examine that for small $b$ deflection angle increases but with the increase of $b$ the deflection angle decreases for fixed $\mu$. So for stable behavior we choose the domain $b \in[1,15]$.

1. Figure (i), illustrate the graphical impact of deflection angle w.r.t $b$ by varying $K$. For negative $K$, we obtain locally hyperbolic behavior but for $K=0$ the behavior is locally flat. If there is small change in the variation of $\mathrm{K}$, deflection angle is exponentially decreasing.

2. Figure (ii), shows that with the increase of $K$ the deflection angle gradually decreases and then goes to positive infinity. We obtain physical stable behavior just for $K \geq 0.5$.

\section{PHOTON LENSING IN A PLASMA MEDIUM}

In this section, we investigate the effect of plasma medium on the photon lensing by asymptotically flat black hole in Horndeski theory. The refractive index for flat black hole is stated as follows [41],

$$
n(r)=\sqrt{1-\frac{\omega_{e}^{2}}{\omega_{\infty}^{2}} H(r)}
$$

then, the corresponding optical metric illustrated as

$$
d \sigma^{2}=g_{j k}^{o p t} d x^{j} d x^{k}=\frac{n^{2}(r)}{H(r)}\left[\frac{60 K}{H(r)} d r^{2}+r^{2} d \varphi^{2}\right] .
$$

where the metric function of the above optical metric is given as

$$
H(r)=60 K^{2}-\frac{\mu}{r}
$$


The optical Gaussian curvature for the above optical metric (18) is calculated by using Eq. 8 as follows

$$
\begin{aligned}
\mathcal{K}= & -\frac{\mu K}{r^{3}}+\frac{\mu^{2}}{80 K r^{4}}+\frac{90 \mu K^{3}}{r^{3}} \frac{\omega_{e}^{2}}{\omega_{\infty}^{2}}-\frac{9 \mu^{2} K}{4 r^{4}} \frac{\omega_{e}^{2}}{\omega_{\infty}^{2}}-\frac{3 \mu K}{r^{4}} \\
& \left(60 K^{2} r-\mu\right) \frac{\omega_{e}^{2}}{\omega_{\infty}^{2}}+\frac{\mu^{3}}{80 K r^{5}} \frac{\omega_{e}^{2}}{\omega_{\infty}^{2}}+\frac{3 \mu^{2}\left(60 K^{2} r-\mu\right)}{80 K r^{5}} \frac{\omega_{e}^{2}}{\omega_{\infty}^{2}} .
\end{aligned}
$$

Hence we get differently which goes to $\alpha$ :

$$
\left.\lim _{x \rightarrow \infty} \hat{k}_{g} \frac{d \tilde{\sigma}}{d \varphi}\right|_{C_{R}}=\alpha .
$$

We use straight line approximation $r=b / \sin \varphi$, for the limit $x \rightarrow \infty$, then GBT stated as [41]

$$
\left.\lim _{x \rightarrow \infty} \int_{0}^{\pi+\tilde{\alpha}}\left[\hat{k}_{g} \frac{d \tilde{\sigma}}{d \varphi}\right]\right|_{C_{R}} d \varphi=\pi-\lim _{x \rightarrow \infty} \int_{0}^{\pi} \int_{b / \sin \varphi}^{x} \mathcal{K} d S .
$$

After simplification, we obtain

$$
\tilde{\alpha} \simeq \frac{\mu^{2} \pi}{320 b^{2} K}+\frac{\mu^{3}}{90 b^{3} K} \frac{\omega_{e}^{2}}{\omega_{\infty}^{2}}+\frac{2 \mu K}{b}-\frac{3 \mu^{2} K \pi}{4 b^{2}} \frac{\omega_{e}^{2}}{\omega_{\infty}^{2}}+\frac{180 \mu K^{3}}{b} \frac{\omega_{e}^{2}}{\omega_{\infty}^{2}}+\mathcal{O}\left(\mu^{4}, K^{4}\right) .
$$

The above results shows that the photon rays are moving in a medium of homogeneous plasma.

\section{A. Graphical Analysis}

This section is focused to investigate the graphical effect of deflection angle $\tilde{\alpha}$ on asymptotically flat BHs in a plasma medium. Further, we exemplify the physical implications of these graphs to analyze the effect of curvature constant $K, \frac{\omega_{e}}{\omega_{\infty}}$ and impact parameter $b$ on deflection angle by analyzing the stable and unstable state of $\mathrm{BH}$.

\section{Deflection angle versus Impact parameter $b$}

This subsection offers the examination of deflection angle $\tilde{\alpha}$ w.r.t impact parameter $b$ for different ranges of curvature constant $K, \frac{\omega_{e}}{\omega_{\infty}}$, for fixed $\mu=2$. For simplicity, here we suppose $\frac{\omega_{e}}{\omega_{\infty}}=\eta$.
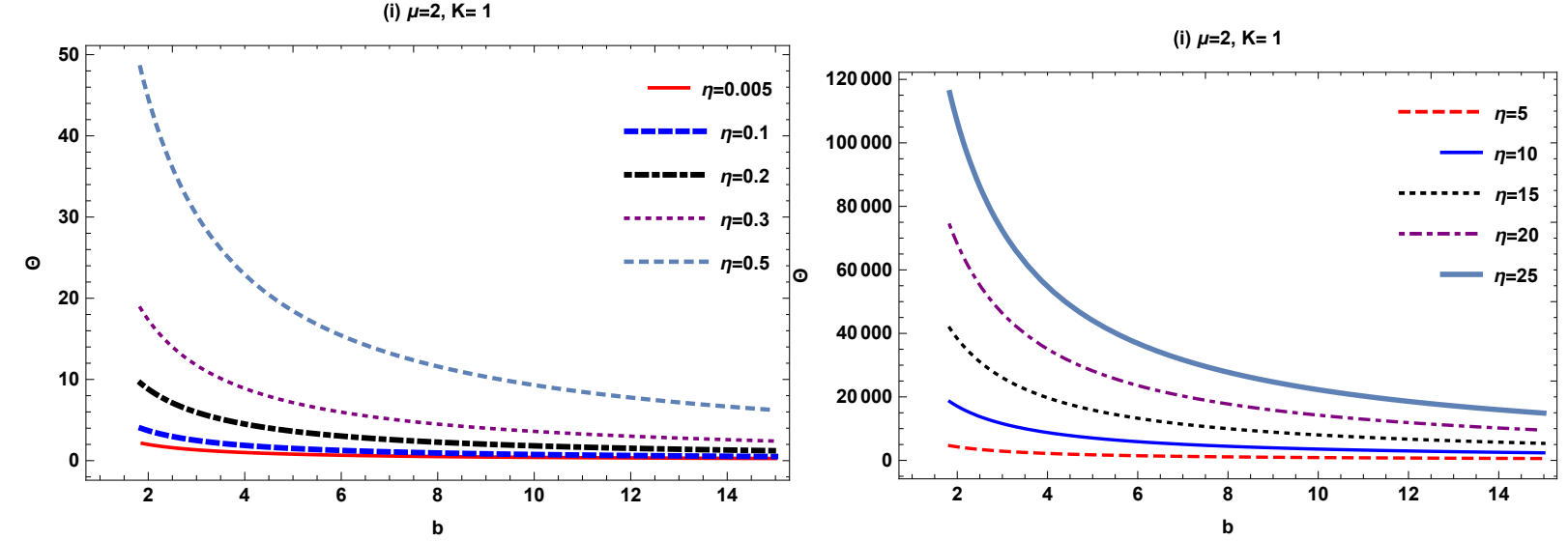

Figure 2: Relation between $\tilde{\alpha}$ and impact parameter $b$.

- Figure 2 depicts the influence of $\tilde{\alpha}$ w.r.t $b$ for varying $\eta$ and for fixed $\mu=2$ and $K=1$.

1. In figure (i), represents the behavior of $\tilde{\alpha}$ w.r.t $b$ for small variation of $\eta$. For $\eta=0.005$ the deflection angle decreases, it is observed that the behavior is same for $\eta=0.005 \rightarrow 0.01$ and $0.01 \rightarrow 0.1$ there is small change in deflection angle but greater than 0.1 the deflection angle actually increase.

2. In figure (ii), shows that the deflection angle gradually increase by increasing $\eta$. 


\section{CONCLUSION}

In this paper, we accomplished an extensive analysis of deflection angle of light by asymptotically flat $\mathrm{BH}$ in the background of Horndeski theory in weak field approximation. In this regard, we employ the optical geometry of asymptotically flat BH in Horndeski theory. Thenceforth, we have utilized the GBT by using straight line approximation and compute the deflection angle in leading order terms. The obtained deflection angle is evaluated by integrating a domain outside the impact parameter, that depict the globally impact of gravitational lensing. Additionally, we have found the deflection angle of photon by asymptotically flat BH in plasma medium. For couple case, we have analyzed graphically the influence of impact parameter, curvature constant and plasma term on deflection angle of photon by asymptotically flat $\mathrm{BH}$ in the context of Horndeski theory. We have examined that the proposed deflection angle increases by decreasing the impact parameter also the mass term $\mu$ decreases the deflection angle, on the contrary, with the increase of curvature constant the deflection angle gradually decreasing. Moreover, if we disregard the impact of plasma medium $\left(\frac{\omega_{e}}{\omega_{\infty}} \rightarrow 0\right)$, in the following equation

$$
\tilde{\alpha} \simeq \frac{\mu^{2} \pi}{320 b^{2} K}+\frac{\mu^{3}}{90 b^{3} K} \frac{\omega_{e}^{2}}{\omega_{\infty}^{2}}+\frac{2 \mu K}{b}-\frac{3 \mu^{2} K \pi}{4 b^{2}} \frac{\omega_{e}^{2}}{\omega_{\infty}^{2}}+\frac{180 \mu K^{3}}{b} \frac{\omega_{e}^{2}}{\omega_{\infty}^{2}}+\mathcal{O}\left(\mu^{4}, K^{4}\right),
$$

we obtain the weak deflection angle for non-plasma medium case:

$$
\tilde{\alpha}=\frac{2 K \mu}{b}+\frac{\mu^{2} \pi}{320 K b^{2}}+\mathcal{O}\left(\mu^{3}\right)
$$

The observations that follow from the Horndeski theory and its mathematical implications include determining observables such as angular positions, separation, magnification and fluxes: a case study of astrophysical applications for Sagittarius $A^{*}$ and M87 can be found in [11]. Additionally, distinct researches include linear Horndeski theories to study dark energy and gravitational waves [83], and Horndeski gravity to study dark matter [84]. Our results can be extrapolated to correct for quantum effects while determining the observables, thus, increasing precision - however, the resulting calculations to attain it are beyond the scope of this work.

\section{Acknowledgments}

This work was supported by Comisión Nacional de Ciencias y Tecnología of Chile through FONDECYT Grant $N^{\circ}$ 3170035 (A. Ö.).

[1] M. Bartelmann and P. Schneider, Phys. Rept. 340, 291 (2001)

[2] T. Clifton, P. G. Ferreira, A. Padilla and C. Skordis, Phys. Rept. 513, 1 (2012)

[3] P. A. R. Ade et al. [Planck Collaboration], Astron. Astrophys. 594, A17 (2016)

[4] S. Capozziello and M. De Laurentis, Phys. Rept. 509, 167 (2011)

[5] R. V. Wagoner, Phys. Rev. D 1, 3209 (1970).

[6] D. Blas, O. Pujolas and S. Sibiryakov, Phys. Rev. Lett. 104, 181302 (2010)

[7] G. W. Horndeski, Int. J. Theor. Phys. 10, 363 (1974).

[8] C. A. R. Herdeiro and E. Radu, Int. J. Mod. Phys. D 24, no. 09, 1542014 (2015)

[9] A. Anabalon, A. Cisterna and J. Oliva, Phys. Rev. D 89, 084050 (2014)

[10] E. Babichev, C. Charmousis and A. Lehbel, JCAP 1704, no. 04, 027 (2017).

[11] J. Badia and E. F. Eiroa, Eur. Phys. J. C 77, no. 11, 779 (2017).

[12] D. C. Latimer, Phys. Rev. D 88, 063517 (2013)

[13] T. Elghozi, N. E. Mavromatos and M. Sakellariadou, Eur. Phys. J. C 77, no. 7, 445 (2017)

[14] B. Ahmedov, B. Turimov, Z. Stuchlk and A. Tursunov, Int. J. Mod. Phys. Conf. Ser. 49, 1960018 (2019).

[15] B. Turimov, B. Ahmedov, A. Abdujabbarov and C. Bambi, arXiv:1802.03293 [gr-qc].

[16] A. Abdujabbarov, B. Ahmedov, N. Dadhich and F. Atamurotov, Phys. Rev. D 96, no. 8, 084017 (2017).

[17] J. Schee, Z. Stuchlk, B. Ahmedov, A. Abdujabbarov and B. Toshmatov, Int. J. Mod. Phys. D 26, no. 5, 1741011 (2017),

[18] H. Ghaffarnejad, M. Amirmojahedi and H. Niad, Adv. High Energy Phys. 2018, 3067272 (2018)

[19] A. B. Aazami, C. R. Keeton and A. O. Petters, J. Math. Phys. 52, 102501 (2011)

[20] K. S. Virbhadra and C. R. Keeton, Phys. Rev. D 77, 124014 (2008)

[21] C. R. Keeton and A. O. Petters, Phys. Rev. D 73, 044024 (2006)

[22] C. R. Keeton and A. O. Petters, Phys. Rev. D 72, 104006 (2005) 
[23] A. Bhadra, Phys. Rev. D 67, 103009 (2003)

[24] W. G. Cao and Y. Xie, Eur. Phys. J. C 78, no. 3, 191 (2018).

[25] Y. K. Lim and Q. h. Wang, Phys. Rev. D 95, no. 2, 024004 (2017)

[26] J. Sultana, JCAP 1304, 048 (2013).

[27] P. Fleury, J. Larena and J. P. Uzan, Phys. Rev. Lett. 119, no. 19, 191101 (2017)

[28] R. Whisker, Phys. Rev. D 71, 064004 (2005)

[29] S. b. Chen and J. 1. Jing, Phys. Rev. D 80, 024036 (2009)

[30] E. F. Eiroa, G. E. Romero and D. F. Torres, Phys. Rev. D 66, 024010 (2002)

[31] C. Y. Wang, Y. F. Shen and Y. Xie, JCAP 1904, 022 (2019)

[32] S. Mao and B. Paczynski, Astrophys. J. 374, L37 (1991).

[33] V. Bozza, Phys. Rev. D 66, 103001 (2002)

[34] M. Sharif and S. Iftikhar, Astrophys. Space Sci. 357, no. 1, 85 (2015).

[35] K. S. Virbhadra and G. F. R. Ellis, Phys. Rev. D 65, 103004 (2002).

[36] O. Kasikci and C. Deliduman, Phys. Rev. D 100, no. 2, 024019 (2019)

[37] R. Zhang and J. Jing, Eur. Phys. J. C 78, no. 10, 796 (2018).

[38] G. W. Gibbons and M. C. Werner, Class. Quant. Grav. 25, 235009 (2008).

[39] M. C. Werner, Gen. Relat. gravit. 44, 3047 (2012).

[40] A. Ishihara, Y. Suzuki, T. Ono, T. Kitamura and H. Asada, Phys. Rev. D 94, no. 8, 084015 (2016)

[41] G. Crisnejo and E. Gallo, Phys. Rev. D 97, no. 12, 124016 (2018)

[42] A. Ishihara, Y. Suzuki, T. Ono and H. Asada, Phys. Rev. D 95, no. 4, 044017 (2017).

[43] H. Arakida, Gen. Rel. Grav. 50, no. 5, 48 (2018).

[44] K. Jusufi, M.C. Werner, A. Banerjee, A. Ovgun, Phys. Rev. D 95(10), 104012 (2017).

[45] I. Sakalli, A. Ovgun, EPL 118(6), 60006 (2017).

[46] K. Jusufi, A. Ovgun, Phys. Rev. D 97(2), 024042 (2018).

[47] W. Javed, R. Babar, A. Ovgun, Phys. Rev. D 99(8), 084012 (2019).

[48] A. Övgün, Phys. Rev. D 99, 104075 (2019).

[49] T. Ono, A. Ishihara and H. Asada, Phys. Rev. D 98, 044047 (2018).

[50] T. Ono, A. Ishihara and H. Asada, Phys. Rev. D 96, 104037 (2017).

[51] K. Jusufi, A. Övgün and A. Banerjee, Phys. Rev. D 96, n084036 (2017) Addendum: [Phys. Rev. D 96, 089904 (2017)].

[52] A. Övgün, K. Jusufi and I. Sakalli, Phys. Rev. D 99, 024042 (2019).

[53] K. Jusufi and A. Övgün, Int. J. Geom. Meth. Mod. Phys. (2019) 1950116.

[54] K. Jusufi, M. C. Werner, A. Banerjee, and A. Övgün, Phys. Rev. D 95, no. 10, 104012 (2017).

[55] K. Jusufi, I. Sakalli, and A. Övgün, Phys. Rev. D 96, no. 2, 024040 (2017).

[56] T. Ono, A. Ishihara, and H. Asada, Phys. Rev. D 99, no. 12, 124030 (2019).

[57] K. Jusufi, A. Övgün, A. Banerjee and I. Sakalli, Eur. Phys. J. Plus 134, no. 9, 428 (2019).

[58] Z. Li and T. Zhou, Phys. Rev. D 101, 044043 (2020).

[59] Z. Li, G. He and T. Zhou, Phys. Rev. D 101, no. 4, 044001 (2020).

[60] K. de Leon and I. Vega, Phys. Rev. D 99, no. 12, 124007 (2019)

[61] G. Crisnejo, E. Gallo, and A. Rogers, Phys. Rev. D 99, 124001 (2019).

[62] G. Crisnejo, E. Gallo, and J. R. Villanueva, Phys. Rev. D 100, no. 4, 044006 (2019).

[63] A. Övgün, G. Gyulchev, and K. Jusufi, Annals Phys. 406, 152 (2019).

[64] K. Jusufi and A. Övgün, Phys. Rev. D 97, 064030 (2018).

[65] K. Jusufi, A. Övgün, J. Saavedra, Y. Vasquez, and P. A. Gonzalez, Phys. Rev. D 97, 124024 (2018).

[66] A. Övgün, Phys. Rev. D 98, 044033 (2018).

[67] A. Övgün, K. Jusufi, and I. Sakalli, Annals Phys. 399, 193 (2018).

[68] A. Övgün, Universe 5, 115 (2019).

[69] A. Övgün, I. Sakalli, and J. Saavedra, Annals Phys. 411, 167978 (2019).

[70] A. Övgün, I. Sakalli, and J. Saavedra, JCAP 1810, 041 (2018).

[71] W. Javed, R. Babar, and A. Övgün, Phys. Rev. D 100, 104032 (2019).

[72] W. Javed, J. Abbas, and A. Övgün, Eur. Phys. J. C 79, no. 8, 694 (2019).

[73] W. Javed, J. Abbas and A. Övgün, Annals Phys. 418, 168183 (2020).

[74] W. Javed, j. Abbas and A. Övgün, Phys. Rev. D 100, no. 4, 044052 (2019).

[75] Y. Kumaran and A. Övgün, Chin. Phys. C 44, 025101 (2020).

[76] W. Javed, M. B. Khadim and A. Övgün, Eur. Phys. J. Plus 135, no.7, 595 (2020).

[77] A. Övgün and I. Sakalli, [arXiv:2005.00982 [gr-qc]].

[78] Z. Li, G. Zhang and A. Övgün, Phys. Rev. D 101, no.12, 124058 (2020).

[79] W. Javed, M. B. Khadim, A. Övgün and J. Abbas, Eur. Phys. J. Plus 135, no.3, 314 (2020).

[80] Z. Li and A. Övgün, Phys. Rev. D 101, no.2, 024040 (2020).

[81] W. Javed, A. Hamza and A. Övgün, Phys. Rev. D 101, no.10, 103521 (2020).

[82] A. Övgün, I. Sakalli and J. Saavedra, arXiv:1908.04261 [gr-qc] (Accepted to publish in CPC).

[83] L. Perenon and H. Velten, Universe 5 (2019) no.6, 138 doi:10.3390/universe5060138

[84] A. Diez-Tejedor, F. Flores and G. Niz, Phys. Rev. D 97 (2018) no.12, 123524 doi:10.1103/PhysRevD.97.123524 